Abridged Edition-, 4, 23 (1966)

3) Onda, J., Sada, E., and Murase, Y.: AIChEJ, 5, 235 (1959)

4) Onda, K., Sada, E., Kido, C., and Tanaka, A.: Kagaku

Kōgaku (Japan) -Abridged Edition-, 1, 50 (1963)
5) Onda, K., Takeuchi, H., and Okamoto, Y.: J. Chem. Eng. Japan, 1, $56(1968)$

6) Semmelbauer, R.: Chem. Eng. Sci., 22, 1237 (1967)

\title{
AGE DISTRIBUTIONS OF SUSPENDED SOLID PARTICLES IN A BUBBLE COLUMN*
}

\author{
YOKO YAMANAKA, TSUNEO SEKIZAWA** AND \\ HIROSHI KUBOTA \\ Department of Chemical Engineering, Tokyo \\ Institute of Technology, Tokyo
}

\begin{abstract}
On the basis of a one-dimensional dispersion model, which was applied by Imafuku et al. to interpret the behavior of suspended solid particles in a bubble column, a procedure for obtaining the age distribution and the spatial age distribution of the particles in a continuously operating bubble column was developed.
\end{abstract}

Imafuku, Wang, Koide and Kubota ${ }^{1)}$ observed experimentally the behavior of suspended solid particles in a bubble column. The data obtained were interpreted by a one-dimensional dispersion model.

Consider the continuous operation of a gas-liquid reaction which is catalyzed by suspended solid particles. The solid particles will be fed into and withdrawn from the column continuously. If a decay in activity of the catalyst particles related to its retention time exists, information about the age distribution and the spatial age distribution are essential to predict reactor performance in this system, as well as information about the profile of solid concentration.

It is difficult to obtain these age distributions experimentally. But when one has correct information about the dispersion coefficient and the settling velocity of solid particles in the column, and when the mathematical model established by experiment at steady state is

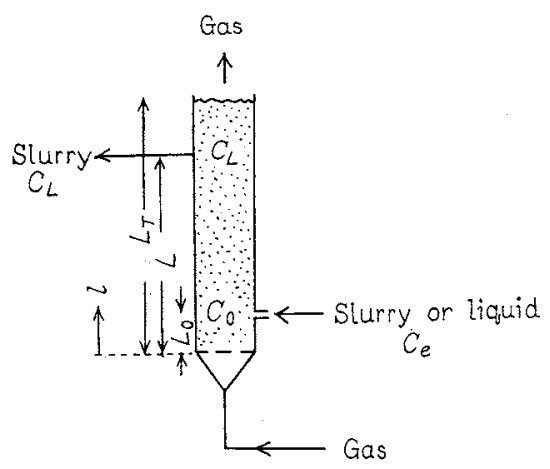

Fig. I Scheme of continuous operation of the bubble column with suspended solid particles

* Received on November 29, 1969

** Nagaoka Technical College, Nagaoka, Niigata consistent enough for extension to transient analysis, these age distributions can be obtained by computational analysis alone.

In the present paper, the procedure for obtaining these age distributions on the basis of a one-dimensional dispersion model, will be discussed.

\section{The One-dimensional Dispersion Model}

According to the one-dimensional dispersion model, mass balance of suspended solid particles in the bubble column, illustrated by Fig. 1, gives the following partial differential equation.

$$
\begin{aligned}
\frac{\partial\{(1-\phi) C\}}{\partial \theta}= & \frac{\partial}{\partial l}\left\{(1-\phi) E_{p} \frac{\partial C}{\partial l}\right\}-u_{l} \frac{\partial C}{\partial l} \\
& +\frac{\partial}{\partial l}\left\{(1-\phi) v_{f}(C) C\right\}
\end{aligned}
$$

Settling velocity, $v_{f}(C)$, is given by ${ }^{1)}$

$$
v_{f}(C)=v_{f 0} \cdot 1.45 \cdot v_{f t}{ }^{-0.35}
$$

where $v_{f 0}=v_{f t} / F(\varepsilon)$ and $F(\varepsilon)=\varepsilon^{-4.65}=\left(1-C / \rho_{s}\right)^{-4.65}$. Boundary conditions for Eq. (1) are stated as;

$l=L_{0} ;-E_{p} \frac{\partial C}{\partial l}-v_{f}(C) C+\frac{u_{l}}{1-\phi}\left(C-C_{e}\right)=0$

$l=L_{T}, \quad l=0 ; E_{p} \frac{\partial C}{\partial l}+v_{f}(C) C=0$

An additional condition for Eq. (1), referring to Fig. 1, is given by

$$
0 \leq l<L_{0}, L<l \leq L_{T} ; u_{l}=0
$$

For the convenience of mathematical development of Eq. (1), the normalization of some variables will be made as ;

$$
\begin{aligned}
& \boldsymbol{C}=C / C_{e}{ }^{*}, X=l / L_{T}, X_{L}=L / L_{T}, X_{0}=L_{0} / L_{T}, \\
& \boldsymbol{C}_{e}=C_{e} / C_{e}{ }^{*}
\end{aligned}
$$

Thus $C_{e}$ is unity when slurry is fed at steady-state operation and zero when liquid containing no solid particles is fed. 


\section{Profile of Suspended Solid Concentration at Steady State}

By substituting zero as the time derivative, normalizing by Eq. (6) and integrating once over $X$, Eq. (1) is reduced to

$$
\begin{aligned}
& -(1-\phi) \frac{E_{p}}{L_{T}} \cdot \frac{d \boldsymbol{C}}{d X}+u_{l} \boldsymbol{C} \\
& -(1-\phi) v_{f}\left(C_{e} \cdot \boldsymbol{C}\right) \boldsymbol{C}+C_{1}=0
\end{aligned}
$$

The integration constant $C_{1}$ is obtained by employing Eq. (3) as a boundary condition, and the equation of steady state mass balance is given by

$$
\frac{d \boldsymbol{C}}{d X}=\frac{L_{T} u_{l}\left(\boldsymbol{C}-\boldsymbol{C}_{e}\right)}{E_{p}(1-\phi)}-\frac{L_{T}}{E_{p}} v_{f}\left(C_{\epsilon} \cdot \boldsymbol{C}\right) \boldsymbol{C}
$$

In the region where $u_{l}=0$, i. e. $0 \leq X \leq X_{0}$ and $X_{L} \leq X \leq$ 1 , the first term of the right-hand side of Eq. (8) is omitted and

$$
\frac{d \boldsymbol{C}}{d X}=-\frac{L_{r}}{E_{p}} v_{f}\left(C_{e} \cdot \boldsymbol{C}\right) \boldsymbol{C}
$$

Since there is no accumulation of solid particles within the column, the following boundary condition for Eqs. (8) and (9) can be set.

$$
X=X_{L} ; C=C_{L}=C_{e}=1
$$

These are the fundamental equations which were used by Imafuku et al. ${ }^{13}$ to analyze the behavior of suspended solid particles within the bubble column. It should be noted that Eqs. (8) and (9) are valid even if $E_{p}$ and $\phi$ vary with $X$.

Eqs. (8) and (9) can be integrated numerically to obtain the profile of suspended solid concentration with the boundary condition of Eq. (10). Then the holdup of solid particles within the column is obtained by

$$
\bar{C}=\int_{0}^{1}(1-\phi) C d X / \int_{0}^{1}(1-\phi) d X
$$

Average residence time of solid particles is

$$
\bar{\theta}_{\mathrm{sta} .}=\frac{L_{T}}{\mathcal{u}_{l}}\left\{\int_{0}^{1}(1-\phi) d X\right\} \overline{\boldsymbol{C}}_{\mathrm{std}}
$$

\section{Age Distribution Functions of Solid Particles}

The age distribution functions of suspended solid particles within the column will be obtained by analyzing the dynamic response of the system, represented by Eqs. $(1) \sim(5)$. Detailed description of deriving age distribution functions is found in Petersen's article ${ }^{3)}$. The dynamic response will be obtained by examining the fractional content of tagged particles, say red, as a function of time since an instant when the feed of normal particles, say white, has been replaced by that of the red particles.

Eq. (1) will be solved with the following initial conditions to obtain the fraction of white at any time.

$$
\begin{array}{cc}
\theta=0, \quad 0 \leq X \leq 1 ; & ; C_{X}=C_{X, \text { std }} \\
\theta \geq 0 & ; C_{e}=0
\end{array}
$$

where $C_{X \text {,std. }}$. can be obtained as a steady state solution mentioned in the foregoing section.

Based on the definition of age distribution functions, it is obvious that the settling velocity of solid particles should be equivalent to that of the steady state conditions, at any point in the column:

$$
v_{f}(C)_{X, \theta}=v_{f}\left(C_{X: \text { st. }} .\right)
$$

The transient profiles of solid concentration obtained by solving Eqs. (1), (13) and (14) give the fraction of the white.

The exit age distribution, $E(\tau)$, the internal age distribution function, $I(\tau)$, and the holdback ${ }^{2)}$ of particles, $H$, will be given by the transient concentration profiles of solid particles:

$$
\begin{aligned}
& E(\tau)=-\left\{\frac{\partial C}{\partial\left(\theta / \bar{\theta}_{\text {std. }}\right)}\right\}_{X_{L}, \tau .} \\
& I(\tau)=\frac{\boldsymbol{C}\left(X_{L, \tau}\right)}{\boldsymbol{C}\left(\bar{X}_{L, \mathrm{std} .}\right)}=\boldsymbol{C}\left(X_{L}, \tau\right)=1-\int_{0}^{1} E(\tau) d \tau \\
& H=1-\int_{0}^{1} I(\tau) d \tau=\overline{\boldsymbol{C}}_{\mathrm{L}} / \overline{\boldsymbol{C}}_{\text {std. }}
\end{aligned}
$$

The spatial age distribution functions $\phi$ and $\psi^{\prime}$ are defined as follows: $\phi(X, \tau)$ is the fraction of the solid particles at any axial distance, $X$, having resided for a time equal to or less than $\tau$ in the column. $\phi^{\prime}(X, \tau) d \tau$ is the fraction of solid particles at a point $X$ having age between $\tau$ and $\tau+d \tau$. These are given by dynamic response of red particles.

$$
\begin{aligned}
& \phi(X, \tau)=\left(\boldsymbol{C}_{X, \text { std. }}-\boldsymbol{C}_{\boldsymbol{X}, \tau}\right) / \boldsymbol{C}_{\boldsymbol{X} \text {, std. }} \\
& \phi^{\prime}(X, \tau)=\frac{\partial \phi(X, \tau)}{\partial \tau}=\frac{-1}{\boldsymbol{C}_{\boldsymbol{X}, \mathrm{std}}}\left\{\frac{\partial \boldsymbol{C}_{X}}{\partial\left(\theta / \bar{\theta}_{\text {std. }}\right)}\right\}_{\tau}
\end{aligned}
$$

The following dynamic responses are illustratively obtained under a restricted condition of $X_{0}=0$ and $X_{L}=1$ for the facility of finite difference approximation.

Because of high nonlinearity of Eq. (1) with Eq. (2), it is apparent that Eq. (1) should be solved numerically by all means. The partial differential equation (1) was approximated as simultaneous ordinary differential equations referred to any point along the axis of the column, after replacing the spatial derivatives by finite difference terms.

The height of the column, $X_{L}=1$, was divided into $n$ spatial increments, $\Delta X$, then $(n+1)$ grid points were located along the axis. After the spatial derivatives at each grid point were replaced by finite difference equations, $(n+1)$ simultaneous ordinary differential equations were set up, as shown in Table 1, where $i$ indicates a

Table 1 Finite difference approximations to spatial derivatives.

For $i$-th grid point from the bottom, where $1<i<n+1$

$$
\begin{aligned}
& \left(\frac{\partial C}{\partial X}\right)_{i}=\frac{C_{i}-C_{i-1}}{\Delta X} \\
& \left(\frac{\partial^{2} C}{\partial X^{2}}\right)_{i}=\frac{C_{i+1}-2 C_{i}+C_{i-1}}{\Delta X^{2}}
\end{aligned}
$$

For the boundaries, where $i=1$

$$
\begin{aligned}
\left(\frac{\partial \boldsymbol{C}}{\partial X}\right)_{1}= & \frac{L_{T}}{E_{p}}\left\{-v_{f}\left(\boldsymbol{C}_{1, \mathrm{std}}\right) \boldsymbol{C}_{1}+\frac{u_{l}}{1-\phi}\left(\boldsymbol{C}_{1}-\boldsymbol{C}_{e}\right)\right\} \\
\left(\frac{\partial^{2} \boldsymbol{C}}{\partial X^{2}}\right)_{1}= & -\frac{2 L_{T}}{\Delta X_{p}}\left\{-v_{f}\left(\boldsymbol{C}_{1, \mathrm{std} .}\right) \boldsymbol{C}_{1}+\frac{u_{l}}{1-\phi}\left(\boldsymbol{C}_{1}-\boldsymbol{C}_{e}\right)\right\} \\
& +\frac{1}{\Delta X^{2}}\left(-\boldsymbol{C}_{3}+4 \boldsymbol{C}_{2}-3 \boldsymbol{C}_{1}\right)
\end{aligned}
$$

and where $i=n+1$

$$
\begin{aligned}
\left(\frac{\partial \boldsymbol{C}}{\partial X}\right)_{n+1}= & -\frac{L_{T}}{E_{p}} v_{f \cdot}\left(\boldsymbol{C}_{n+1, \mathrm{std} .}\right) \boldsymbol{C}_{n+1} \\
\left(\frac{\partial^{2} \boldsymbol{C}}{\partial X^{2}}\right)_{n+1}= & -\frac{2 L_{T}}{\Delta X E_{p}} v_{f}\left(C_{n+1, \mathrm{std} .}\right) C_{n+1} \\
& +\frac{1}{\Delta X^{2}}\left(\boldsymbol{C}_{n-1}-\boldsymbol{C}_{n+1}\right)
\end{aligned}
$$




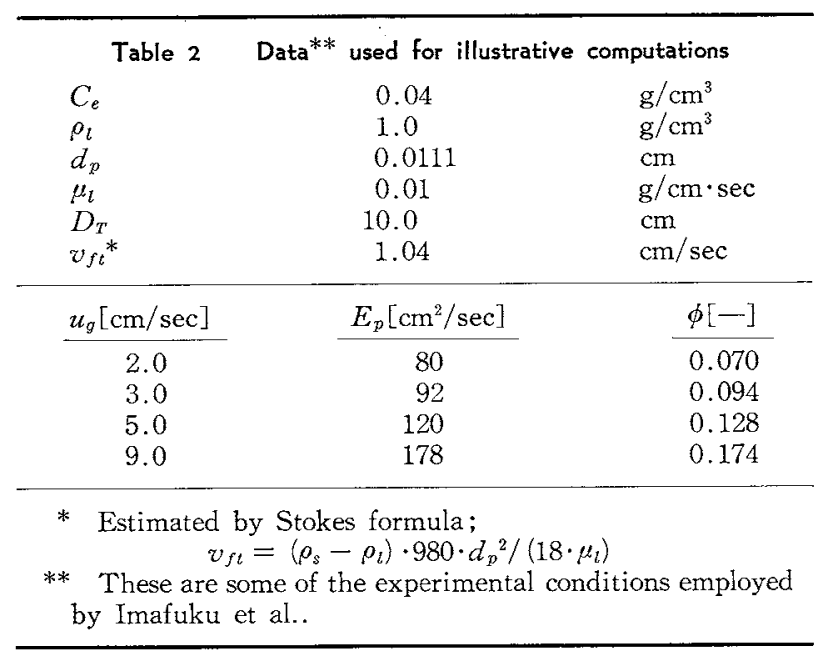

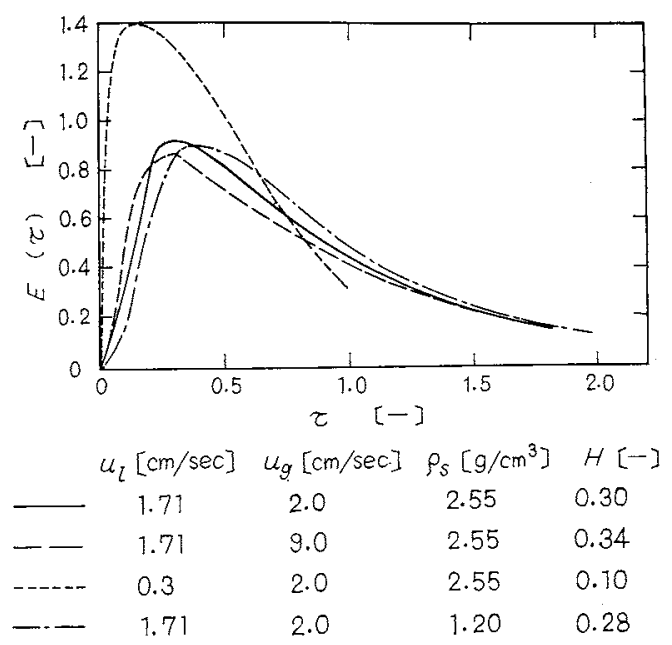

Fig. 2 Residence time distributions of solid particles

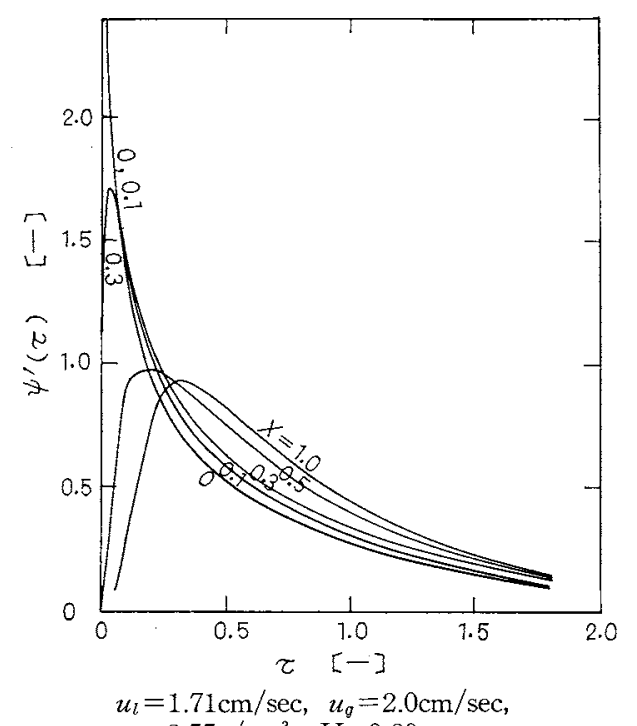

$\rho_{s}=2.55 \mathrm{~g} / \mathrm{cm}^{3}, \quad H=0.30$

Fig. 3 Spatial age distributions of solid particles in the column location of a grid point counted from the bottom.

The numerical solutions at some operating conditions, referred to in Table 2, led to residence time distributions in Fig. 2 and spatial age distributions in Fig. 3. Computing time to integrate Eq. (1) for every operating condition was 20 40 minutes. Time increment for every Runge-Kutta-Gill integration step was monitored so that the value of $C$ at any time might be accurate within 5 digits. It was in the range of $0.125 \sim 1.0 \mathrm{sec}$.

\section{Acknowledgement}

The authors are grateful to Miss H. Shibuya, Mr. M. Hagihara and Mr. S. Furusaki, Mitsui Toatsu Chemicals, Inc., for help on machine computation.

\section{Nomenclature}

$C=$ concentration of solid particles in slurry $\quad\left[\mathrm{g} / \mathrm{cm}^{3}\right]$ $C_{e}=$ concentration of solid particles in feed $\quad\left[\mathrm{g} / \mathrm{cm}^{3}\right]$ $C_{e}{ }^{*}=$ concentration of solid particles in feed slurry at steady state operation

$\left[\mathrm{g} / \mathrm{cm}^{3}\right]$

$\boldsymbol{C}=$ normalized concentration of solid particles, $C / C_{e}{ }^{*}[-]$

$\overline{\boldsymbol{C}}=$ normalized average concentration of solid particles

$d_{p}=$ diameter of a solid particle

$D_{T}=$ diameter of a bubble column

$E_{p}=$ dispersion coefficient of solid particles $\quad\left[\mathrm{cm}^{2} / \mathrm{sec}\right]$

$H=$ holdback of solid particles particles

$i=$ order of finite difference point from the bottom [-]

$l=$ axial height from the bottom $[\mathrm{cm}]$

$L_{0}=$ height of slurry or liquid inlet from the bottom $[\mathrm{cm}]$

$L=$ height of slurry outlet from the bottom $[\mathrm{cm}]$

$L_{T}=$ height of bubble column [cm]

$n=$ number of axial finite difference unit $[-]$

$u_{g}=$ superficial gas velocity $\quad[\mathrm{cm} / \mathrm{sec}]$

$u_{l}=$ superficial velocity of slurry or liquid $[\mathrm{cm} / \mathrm{sec}]$

$v_{f}=$ settling velocity of solid particles $[\mathrm{cm} / \mathrm{sec}]$

$v_{f 0}=$ settling velocity of solid particles in stagnant liquid

$[\mathrm{cm} / \mathrm{sec}]$

$v_{f t}=$ settling velocity of a single particle in stagnant liquid

$X=$ normalized axial height from the bottom, $l / L_{T}{ }^{[\mathrm{cm} / \mathrm{sec}]}$

Greek letters

$\varepsilon=$ void fraction of solid particles in liquid

$\dot{\phi}=$ gas holdup

$\rho_{l}=$ density of liquid

$\mu_{t}=$ viscosity of liquid

$\theta=$ time

$\bar{\theta}_{s}=$ average residence time of solid particles

$\tau=$ normalized age of solid particles in the column $[-]$

Subscripts

$e=$ slurry or liquid inlet

$L=$ slurry outlet

std. = steady state

$i=$ finite difference point

\section{Literature cited}

1) Imafuku, K., Wang, T. Y., Koide, K. and H, Kubota: J. Chem. Eng. Japan, 1, 153 (1968)

2) Danckwerts, P. V.: Chem. Eng. Sci., 2, 1 (1953)

3) Petersen, E. E. : "Chemical Reaction Analysis", Prentice Hall, 1965 